\title{
Human Thermal Comfort Study Based on Average Skin Temperature
}

\author{
Yalong Yang ${ }^{1,2, a}$, Bao Xie ${ }^{1,2, b}$, Qiansheng Fang ${ }^{1,2, c^{*}}$, Mingyue Wang ${ }^{1,2, d}$, Wenmiao Wu ${ }^{1,2, e}$ \\ ${ }^{1}$ Anhui Key Laboratory of Intelligent Building, Anhui Jianzhu University, Hefei, 230022, China \\ ${ }^{2}$ School of Electronic and Information Engineering, Anhui Jianzhu University, Hefei, 230022, China \\ ayangyl@ahjzu.edu.cn, b1246273248@qq.com, ${ }^{\mathrm{a}} \mathrm{qsfang} @$ sohu.com, ${ }^{\mathrm{d}} 754476274 @ q q . c o m$, \\ e997180694@qq.com
}

Keywords: Average skin temperature, thermal comfort, Fisher discriminant method, evaluating model

\begin{abstract}
As an important evaluation index of indoor environmental quality, human thermal comfort is affected by various factors. As human physiological signal, the skin temperature is closely related to human thermal comfort. To investigate the model's mapping relationship between the skin temperature and the human thermal comfort, the skin temperatures of six parts of human body were chosen as research object, the linear mapping relationship between the average skin temperature and the human thermal comfort was analyzed by using Fisher discriminant method under five different experimental conditions and subjective voting. As a result, the mathematical evaluating model between the average skin temperature and human thermal comfort was obtained based on experiments. The results indicate the average precision of this model is high and the discriminant result is reliable, which provides valuable reference for human thermal comfort modeling under homogeneous environmental condition.
\end{abstract}

\section{Introduction}

Building energy consumption plays an important role in national energy security, social stability and economic sustainable development [1]. With the demands on indoor environment quality becomes higher and higher, building energy consumption has become increasingly prominent. Therefore, under the premise of the building energy consumption, how to improve the indoor environmental quality and the human thermal comfort are the problems needed to be solved.

As an important evaluating indicator for indoor environment, human thermal comfort is used to investigate the building energy consumption. Currently, subjective questionnaires is used to evaluate the human thermal comfort, the indoor thermal comfort state is fed back by setting a series of questions of questionnaire. However, the evaluating results will be easily affected by the age of the subjects, gender, mood swings and the adaptation level to experimental environment, which can't reflect the real thermal comfort. In recent years, to search the thermal comfort mechanism from physiology point of view, physiological parameters was employed to perform thermal comfort research by many researchers, which aims at evaluating the human thermal comfort more objectively $[2,3]$. As an important physiological signal of human body, the skin temperature is one of the major factors to maintain the human thermal comfort balance. In this paper, the distribution of subjective 
voting was analyzed based on the gathered skin temperature of the main parts of human body. Moreover, the influence of thermal environment on the skin temperature of human body was investigated. The average skin temperature was regarded as input, the thermal comfort level was graded by using Fisher discriminant method, then the evaluating model of human thermal comfort by the average skin temperature was established.

\section{Fisher discriminant method}

Generally, two classification methods are used, which include parameter identification method and non parameter identification method [4]. The fundamental idea of Fisher discriminant method lies in projection and simplifying of multidimensional problems $[5,6,7]$. In this paper, the skin temperature of forehead (X1), skin temperature of chest (X2), skin temperature of calf (X3), skin temperature of thigh (X4), skin temperature of back (X5) and skin temperature of arm (X6) are regarded as input parameters $\mathrm{X}=(\mathrm{X} 1, \mathrm{X} 2, \mathrm{X} 3 \cdots \mathrm{X} 6)$ for Fisher discriminant method. A linear function will be searched to realize one dimension.

$$
g(x)=W^{T} X+w_{0}=\left(w_{1}, w_{2} \cdots w_{6}\right)\left(\begin{array}{l}
x_{1} \\
\cdots \\
x_{6}
\end{array}\right)+w_{0}
$$

In formula (1), $\mathrm{W}$ is the projection direction of $\mathrm{X}$, the projection of original vector in this direction should ensure the minimum distance of the same category of samples, and the largest distance between different categories, that is to say, the projection of skin temperature vector $\mathrm{X}$ under the same category of comfort level is relative centralization, the projection of skin temperature vector $\mathrm{X}$ under the different category of comfort level is relative decentralization. The functions used to evaluate projection direction $\mathrm{W}$ are as shown in formulas (2) and (3):

$$
\begin{aligned}
& J_{F}(W)=\frac{\left(\tilde{m}_{1}-\tilde{m}_{2}\right)^{2}}{\tilde{S}_{1}{ }^{2}+\tilde{S}_{2}{ }^{2}} \\
& W^{*}=S^{-1}{ }_{W}\left(m_{1}-m_{2}\right)
\end{aligned}
$$

Formulas (2) and (3) are the solutions to the optimal normal vector by using Fisher criterion. $m_{1}-m_{2}$ is the difference vector of the average value of the skin temperature in each category of comfort level. $S_{-1}^{W}$ is the inverse matrix of $S_{W}$, if $m_{1}-m_{2}$ is 6 dimensional, $S_{W}$ and $S_{-1}^{W}$ are 6 dimensions too. Then $W^{*}$ is 6 dimensional vector too. Vector $W^{*}$ is the solution to the maximum value of the function $J_{F}(W)$, which is the weight value to the weighted 6 dimensional vectors according to Fisher criterion. In formulas (4) and (5), the discriminant function $w_{0}$ can be determined by the following method, as shown in Formulas (4) and (5):

$$
\begin{aligned}
& w_{0}=-\frac{\tilde{m}_{1}+\tilde{m}_{2}}{2} \\
& \text { or } \\
& w_{0}=-\frac{N_{1} \tilde{m}_{1}+N_{2} \tilde{m}_{2}}{N_{1}+N_{2}}
\end{aligned}
$$


After $\mathrm{S} 1$ is determined, it can be graded according to the following rules, as shown in Formula (6):

$$
\begin{aligned}
& W^{T} X>-w_{0} \rightarrow X \in w_{1} \\
& W^{T} X<-w_{0} \rightarrow X \in w_{2}
\end{aligned}
$$

\section{Experiments and results}

\subsection{Experimental conditions}

The experiment was performed in human thermal comfort laboratory of Anhui Jianzhu University. Totally, 20 subjects were recruited from the undergraduates, which are 23 to 26 years old and health. EquivitalTM EQ-02, Biofeedbackx-pert, skin temperature chip and HADC0191 were used to perform the experiments. To obtain expected heating and cooling effects, underfloor air supply was used.

Fig. 1 is the layout of the human thermal comfort laboratory, the dimension is $8.7 \mathrm{~m} \times 6.4 \mathrm{~m} \times 2.6 \mathrm{~m}$ (length $\times$ width $\times$ height). The dimension of working area is $8.4 \mathrm{~m} \times 6.1 \mathrm{~m} \times 2.4 \mathrm{~m}$ (length $\times$ width $\times$ height).

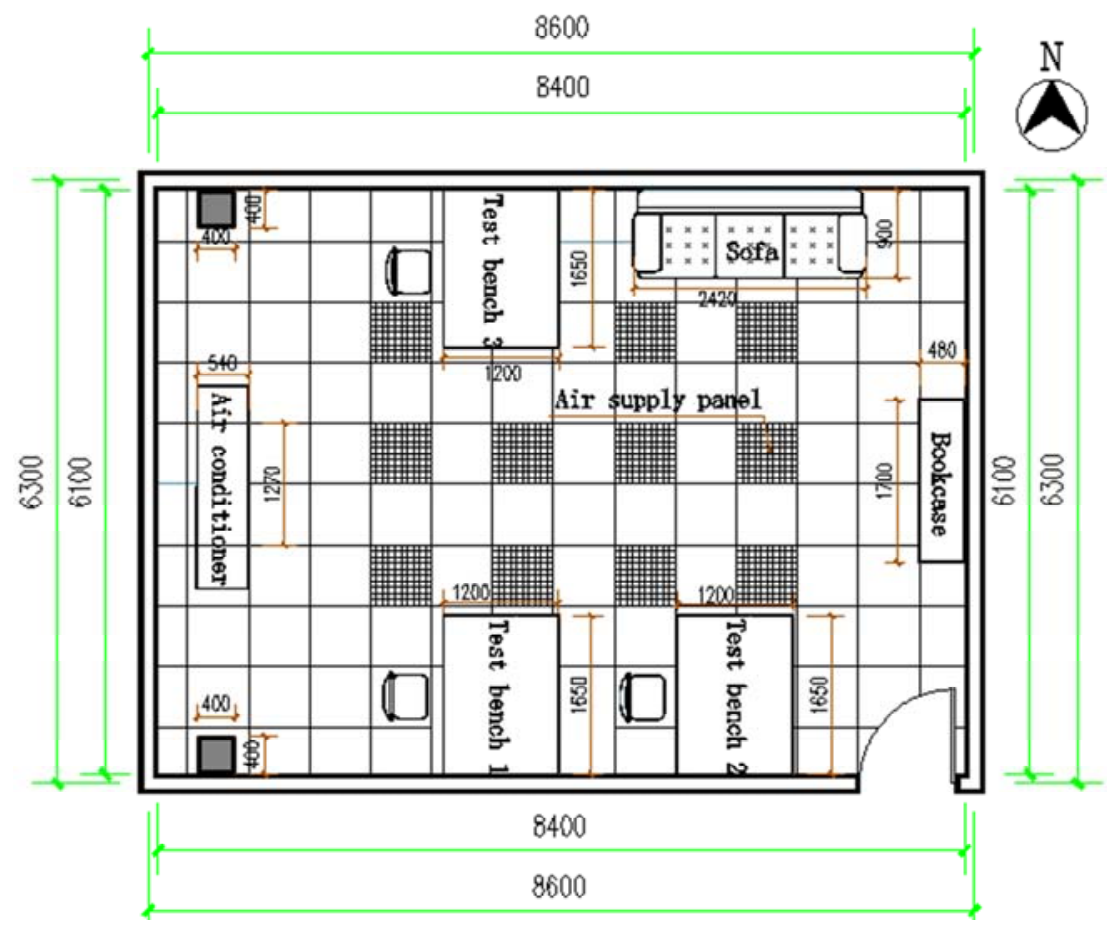

Fig. 1 Layout of human thermal comfort laboratory

There are five working conditions, which include $24^{\circ} \mathrm{C}, 26^{\circ} \mathrm{C}, 28^{\circ} \mathrm{C}, 30^{\circ} \mathrm{C}$ and $32^{\circ} \mathrm{C}, 20$ experiments for each group were performed, which corresponds to 100 person-time. More than 15 minutes are required for each test, the skin temperatures of subjects were collected and they were asked to complete subjective voting. The questionnaire was divided into 3 parts according to Bedford [8] and ASHRAE [9]: matters needing attention of investigation, personal information of subjects, record of thermal environmental parameters and thermal comfort voting. The personal information of subject includes gender, age, height, weight, hometown, average activity level and the local living years. In addition, which also includes experimental time and questionnaire number. As shown in 
table 1, five levels were used to evaluate thermal comfort.

Table 1 The levels of thermal comfort

\begin{tabular}{|c|c|c|c|c|c|}
\hline $\begin{array}{c}\text { Thermal } \\
\text { comfort }\end{array}$ & $\begin{array}{c}\text { Very } \\
\text { comfortable }\end{array}$ & $\begin{array}{c}\text { Acceptable } \\
\text { comfort }\end{array}$ & $\begin{array}{c}\text { Slightly } \\
\text { uncomfortable }\end{array}$ & Discomfort & $\begin{array}{c}\text { Very } \\
\text { uncomfortable }\end{array}$ \\
\hline Ruler & 0 & 1 & 2 & 3 & 4 \\
\hline
\end{tabular}

\subsection{Data acquisition and processing}

In the experiments, the skin temperatures of the subjects' forehead, chest, leg, thigh, back and arm were collected. For each condition, the average values of the 20 groups of skin temperatures were obtained. The skin temperatures distribution are as shown in Figure 2, which indicate the temperature of leg is the lowest but the temperature of forehead is the highest. When the working condition is same and $T=24{ }^{\circ} \mathrm{C}$, the temperature of leg is $31.6^{\circ} \mathrm{C}$, the temperature of forehead is $34.6^{\circ} \mathrm{C}$, the temperature difference is $3^{\circ} \mathrm{C}$. As a result, the temperature difference under low temperature condition is higher than the temperature difference under high temperature condition, the difference is gradually decreased with increasing temperature. Under different working conditions, the temperature of each tested point is increased with the increasing of temperature, but the increasing amplitude is gradually decreased.

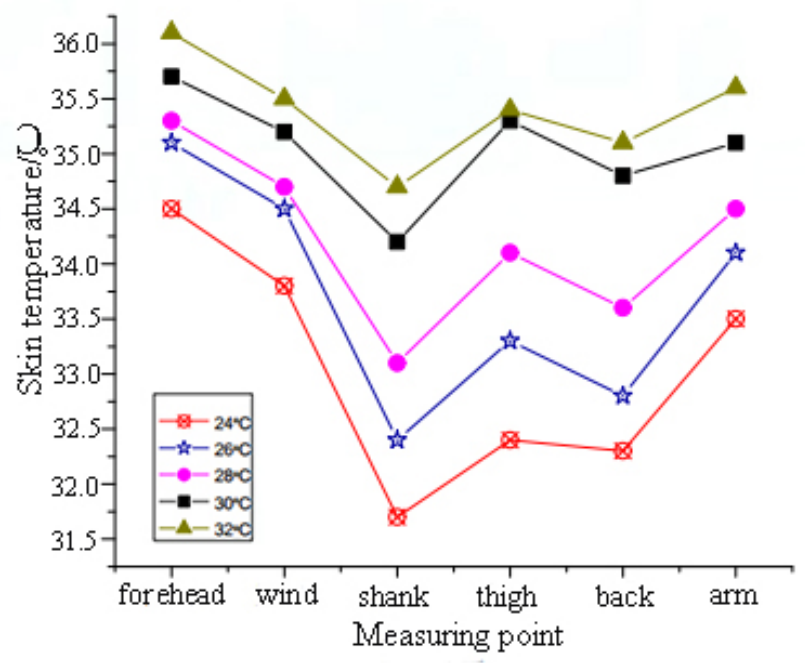

Fig. 2 The skin temperatures distribution under homogeneous environment

Some relative researches indicate more tested points of skin temperature correspond to more real average skin temperature. However, due to restricted experimental conditions, the human tested points are limited. In warm environment, the human skin temperature distribution is average, the average skin temperature can be reflected by 2 to 4 tested points [10]. In neutral environment, the average skin temperature can be reflected by 4 to 8 tested points. Considering human subjective voting, more tested points will result in discomfort but less tested points will result in inaccurate data. Therefore, the calculating formula used to calculate the average skin temperature can be obtained by using six point weighted method, as shown in formula (7).

$$
T_{\text {Skin }}=0.07 A+0.26 B+0.13 C+0.19 D+0.16 E+0.19 F
$$

In formula (7), $\mathrm{A} \sim \mathrm{F}$ represent the forehead, chest, legs, thighs, back and arms respectively. 
After the subjects' subjective voting, the subjective thermal comfort parameters of the human body under different thermal environment were obtained. As shown in Figure 3, when $T=24{ }^{\circ} \mathrm{C}$, the value of TCV did not reach the lowest, the subjects were in a state of discomfort. With the increasing temperature, the value of $T C V$ began to decrease. When $T=26.3{ }^{\circ} \mathrm{C}$, the value of $T C V$ reached the lowest, and then it began to increase slowly. Therefore, the values of the 20 groups of voting were averaged according to different working conditions. Regression was performed based on the average skin temperature and the values of thermal comfort voting, the relationship is as follows:

$$
T C V=1.4134 T_{\text {Skin }}-46.798
$$

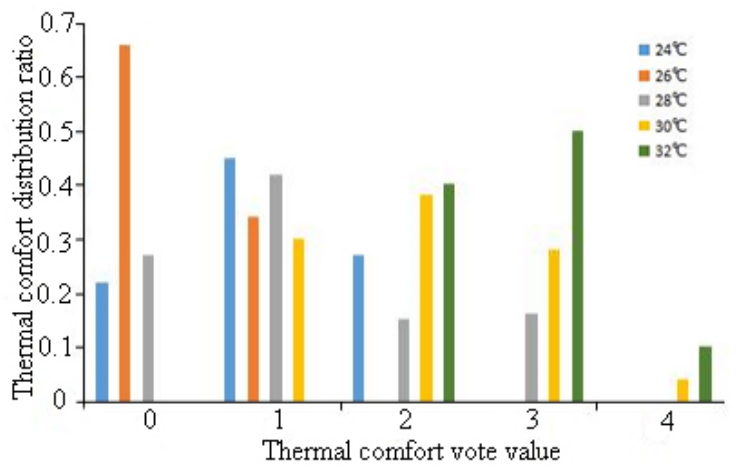

Fig. 3 The voting distribution of thermal comfort

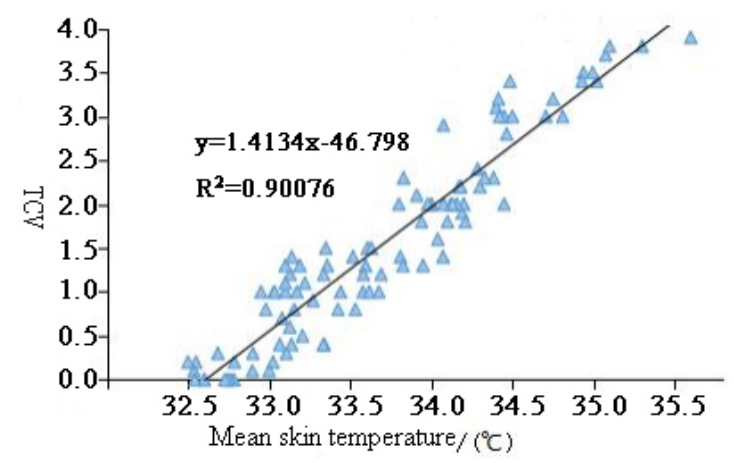

Fig. 4 The relationship between average skin temperature and thermal comfort

As shown in Figure 4, some conclusions can be drawn from the relationship between the average skin temperature and thermal comfort: The values of thermal comfort will be increased with the increasing average skin temperature. The relationship between average skin temperature $T_{\text {skin }}$ and the value $T C V$ of thermal comfort is approximately linear, and the determinant coefficient $\mathrm{R}^{2}$ is 0.90076 .

\subsection{Model establishment}

In this paper, the skin temperatures of forehead, chest, leg, thigh, back and arm are regarded as input. Human thermal comfort levels are regarded as output, and classification was realized by using Fisher discriminant method. According to Fisher criteria, the samples of different categories should have significant differences, so the skin temperature under thermal comfort level needs to be statistically examined. As shown in Table 2.

As shown in Table 2, error samples were rejected, thermal comfort levels can be divided into four categories $(0,1,2 、 3)$. For the samples with thermal comfort levels 0 and 1 , the average skin 
temperatures $u_{0}=33.6^{\circ} \mathrm{C}$ and $u_{1}=33.8^{\circ} \mathrm{C}$, clearly, the average value difference is small. When $T C V=0$, there are only 3 samples.

Therefore, in order to verify the validity of classification, if there is significant difference between the skin temperature of each category should be examined, S-N-K examination of the skin temperatures of various categories was performed, the results are as shown in Table 3.

Table 2 The descriptive statistics of skin temperature

\begin{tabular}{|c|c|c|c|c|c|c|}
\hline \multirow{2}{*}{ TCV } & \multirow{2}{*}{ Sample number } & \multirow{2}{*}{$\begin{array}{c}\text { Average } \\
\text { value }\end{array}$} & \multirow{2}{*}{$\begin{array}{c}\text { Standard } \\
\text { deviation }\end{array}$} & \multirow{2}{*}{$\begin{array}{c}\text { Standard } \\
\text { error }\end{array}$} & \multicolumn{2}{|c|}{$\begin{array}{c}\text { Confidence interval of average } \\
\text { value 95\% }\end{array}$} \\
\cline { 6 - 7 } & & & & & Lower limit & Upper limit \\
\hline 0 & 3 & 33.6 & 0.10 & 0.06 & 33.4 & 33.8 \\
\hline 1 & 21 & 33.8 & 0.23 & 0.05 & 33.7 & 33.9 \\
\hline 2 & 23 & 34.4 & 0.31 & 0.06 & 34.3 & 34.5 \\
\hline 3 & 33 & 35.3 & 0.22 & 0.04 & 35.2 & 35.3 \\
\hline
\end{tabular}

Table 3 Significance examination of skin temperature

\begin{tabular}{|c|c|c|c|c|}
\hline \multirow{2}{*}{ TCV } & \multirow{2}{*}{ Sample number } & \multicolumn{3}{|c|}{ Subset of Alpha $=0.05$} \\
\cline { 3 - 5 } & & 1 & 2 & 3 \\
\hline 0 & 3 & 33.6 & & \\
\hline 1 & 21 & 33.8 & & \\
\hline 2 & 23 & & 34.4 & 35.3 \\
\hline 3 & 33 & & & 1.000 \\
\hline Statistical significance & & 0.073 & 1.000 & \\
\hline
\end{tabular}

As shown in Table 3, the skin temperature of different categories is orderly arranged in longitudinal direction, and there have 3 groups in horizontal direction, $\mathrm{P}$-value between two groups is less than 0.05 , but the P-value in one group is more than 0.05 . For the thermal comfort level between 0 and 1 , it can be seen that there is no significant difference of skin temperatures. Thermal comfort level between 0 and 1 does not significant differences, and there have significant differences of other groups [11].

According to the above analyses, level 0 and level 1 can be combined to meet the Fisher criteria. Thus, the levels of thermal comfort can be divided into three categories, which include netual $(T C V=0$ or $T C V=1)$, hot but a little uncomfortable $(T C V=1)$, hot and uncomfortable $(T C V=3)$.

Fisher discriminant analysis was performed based on the average temperature data of forehead, chest, leg, thigh, back and arm. As shown in Table 4, the two eigen values are $\lambda_{1}=7.883$ and $\lambda_{2}=0.325$. The between-column variance explained by the first discriminant function is $96 \%$, the canonical correlation is 0.942 , and the second discriminant function covers less information.

Table 4 The characteristic values of discriminant function

\begin{tabular}{|c|c|c|c|c|}
\hline Function & Eigen value & Variance percentage & Cumulative percentage & Canonical correlation \\
\hline 1 & 7.883 & 96.0 & 96.0 & 0.942 \\
\hline 2 & 0.325 & 4.0 & 100.0 & 0.495 \\
\hline
\end{tabular}

Because the obtained skin temperature of each tested point was actually measured, therefore, non standardized coefficient was chosen for Fisher discriminant function. As shown in Table 5, which 
indicates the contribution of different input variables to the first two discriminant functions or the first two coordinates.

Table 5 The coefficients of non standardized canonical discriminant function

\begin{tabular}{|c|c|c|}
\hline Coefficient & Discriminant function y1 & Discriminant function y2 \\
\hline T1 & 1.705 & 2.906 \\
\hline T2 & 0.201 & 2.463 \\
\hline T3 & -0.200 & -0.466 \\
\hline T4 & 1.497 & -4.023 \\
\hline T5 & 1.191 & 0.928 \\
\hline T6 & -0.201 & 0.433 \\
\hline (Constant) & -146.107 & -82.423 \\
\hline
\end{tabular}

Table 6 Wik's Lambda Test

\begin{tabular}{|c|c|c|c|c|}
\hline Function test & Lambda & Chi-square & $\begin{array}{c}\text { Degrees of } \\
\text { freedom }\end{array}$ & $\begin{array}{c}\text { Statistical } \\
\text { significance }\end{array}$ \\
\hline 1 through 2 & 0.085 & 183.674 & 12 & 0.000 \\
\hline 2 & 0.755 & 20.956 & 5 & 0.001 \\
\hline
\end{tabular}

Validation of the two Fisher discriminant functions was examined, as shown in Table 6, the results indicate the two discriminant functions are statistically significance, Sig is less than 0.05 . under the significant level of 0.05 , there is significant difference of the average Fisher discriminant function value of different groups, which can be considered effectively [12]. Consequently, the Fisher discriminant function model of human thermal comfort based on skin temperature is obtained:

$$
\begin{aligned}
& y_{1}=1.705 T_{1}+0.201 T_{2}-0.2 T_{3}+1.497 T_{4}+1.191 T_{5}-0.201 T_{6}-146.107 \\
& y_{2}=2.906 T_{1}+2.463 T_{2}-0.466 T_{3}-4.023 T_{4}+0.928 T_{5}+0.433 T_{6}-82.423
\end{aligned}
$$

\subsection{Result analysis}

Fisher discriminant method provides three graphical display methods, which include joint distribution map, individual distribution map and field map. In this paper, the joint distribution map was chosen to analyze and demonstrate the effectiveness of the Fisher discriminant function model. Table 7 is the distribution center of the plan consists of different classes in the first and second discriminant functions.

Table 7 Distribution center of gravity of different categories of samples

\begin{tabular}{|c|c|c|}
\hline \multirow{2}{*}{ TCV } & \multicolumn{2}{|c|}{ Discriminant function } \\
\cline { 2 - 3 } & $\mathrm{y} 1$ & $\mathrm{y} 2$ \\
\hline I & -3.544 & 0.406 \\
\hline II & -0.607 & -0.872 \\
\hline III & 3.001 & 0.273 \\
\hline
\end{tabular}

Three groups of points cluster were constituted by the values of all kinds of discriminant function value in the two-dimensional space. Each cluster has a focus point. As shown in Table 7, The first 
type of center of gravity coordinate is: $(-3.544,0.406)$, the second type of center of gravity coordinate is: $(-0.607,-0.872)$, the third type of center of gravity coordinate is: $(3.001,0.273)$.

In classification, the sample data is substituted into the discriminant function. The coordinate of discriminant points can be found. By comparing the distance between the sample points and all kinds of center of gravity, if the distance is nearest, which belongs to the category [13]. Fig 5 indicates the three categories are separated from one another.

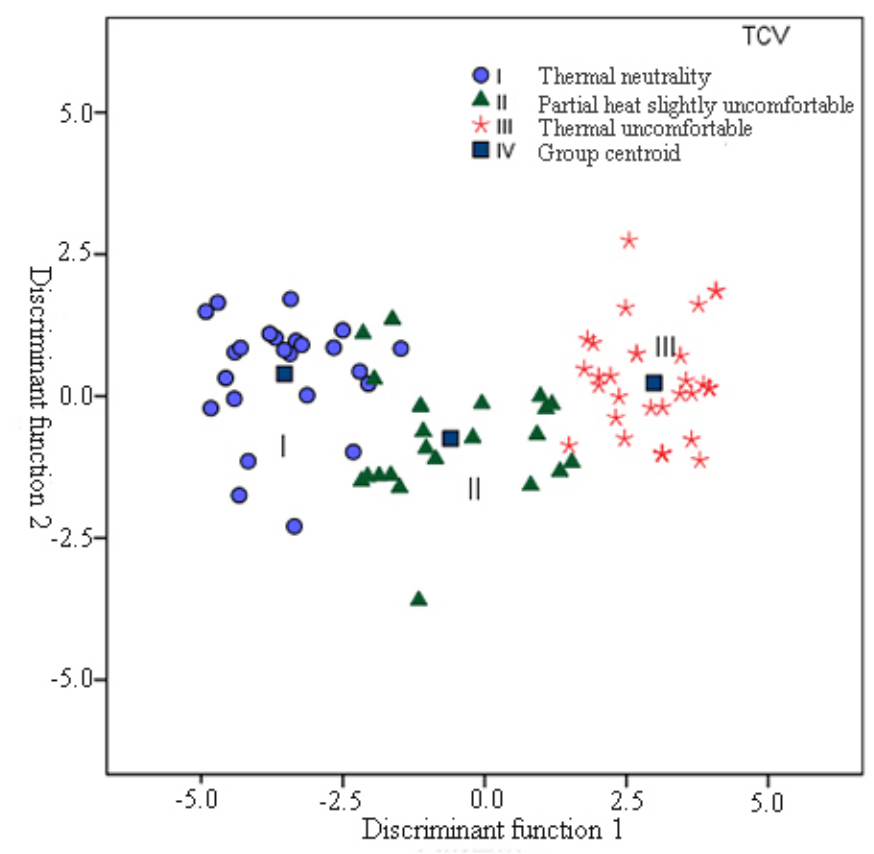

Fig. 5 Joint distribution of center of gravity

Prediction and assessment of the correct rate of Fisher discriminant model was performed. Table 8 indicates the correct rate of class I is $95.8 \%$, the correct rate of class II is $78.3 \%$, the correct rate of class III is $100 \%$, the average correct rate is $92.5 \%$.

Table 8 evaluative information of prediction group

\begin{tabular}{|c|c|c|c|c|c|}
\hline \multirow{2}{*}{ Original values } & \multirow{2}{*}{ TCV } & \multicolumn{3}{|c|}{ Members of prediction group } & \multirow{2}{*}{ Total } \\
\cline { 2 - 6 } & & I & II & III & \\
\hline \multirow{3}{*}{ Differential counting } & I & 23 & 1 & 0 & 24 \\
\cline { 2 - 6 } & II & 3 & 18 & 2 & 23 \\
\cline { 2 - 6 } & III & 0 & 0 & 33 & 33 \\
\hline \multirow{3}{*}{ Correct rate $(\%)$} & I & 95.8 & 4.2 & 0 & 100 \\
\cline { 2 - 6 } & II & 13.0 & 78.3 & 8.7 & 100 \\
\cline { 2 - 6 } & III & 0 & 0 & 100 & 100 \\
\hline
\end{tabular}

\section{Conclusions}

(1) Based on the skin temperatures of forehead, chest, leg, thigh, back and arm, the influence difference of skin temperature of each tested point on the human thermal comfort is objective reflected, which reduce the influence of insignificant index on the validity of evaluation results.

(2) Based on the selected temperatures of six tested points are regarded as evaluation index, the samples can be classified into three types with Fisher discriminant method: Netual, hot but a little uncomfortable, hot and uncomfortable. Moreover, the experimental results indicates the average 
accuracy of this model is higher and the discriminating results are reliable.

\section{Acknowledgements}

This research was funded by the National Natural Science Foundation of China (61300060), the Natural Science Foundation of Anhui Province (1508085QF131) and the Major Project on the Integration of Industry, Education and Research (AJ-CXY-20141220-002).

\section{References}

[1] Jiangyi,Pengchen,Yanda.Technology roadmap of china building energy conversation[J].Building Technology, 2012,17:12-19. (In Chinese)

[2] M.D.P. Garcia-Souto, P. Dabnichki. Skin temperature distribution and thermoregulatory response during prolonged seating $[\mathrm{J}]$. Building and Environment2013, 69:14-21.

[3] Liu,Weiwei; Lian,Zhiwei; Deng,Qihong, est. Objective evaluation index of thermal comfort of human body [J]. Journal of Central South University(Natural Science Edition),2011,02:521-526. (In Chinese)

[4] Peng, Xiaozhi; Wu, Hecheng. Bayes discriminant analysis based on non parametric method [J]. Statistics and decision making, 2015,22:68-70. (In Chinese)

[5] Hong Huang,Hailiang Feng,Chengyu Peng. Complete local Fisher discriminant analysis with Laplacian score ranking for face recognition [J]. Neurocomputing,2012,89.

[6] Chen,Minqiong. Relationship between canonical correlation analysis and Fisher discriminant analysis [J]. Statistics and decision making, 2013,02:21-24. (In Chinese)

[7] Gao, Quanxue; Wang, Qianqian; Huang, Yunfang. Dimensionality Reduction by Integrating Sparse Representation and Fisher Criterion and Its Applications [J]. IEEE Transactions on Image Processing, 2015, 24.

[8] Giaconia Carlo,Orioli Aldo,Di Gangi Alessandra. A correlation linking the predicted mean vote and the mean thermal vote based on an investigation on the human thermal comfort in short-haul domestic flights [J]. Applied ergonomics,2015,48.

[9] A Hussin,E Salleh,HY Chan,et al. The reliability of Predicted Mean Vote model predictions in an air-conditioned mosque during daily prayer times in Malaysia [J]. Architectural Science Review,2015,581.

[10] Sun,Yuming. Experimental study on thermal sensation of human body based on skin temperature [D]. Dalian University of Technology, 2009. (In Chinese)

[11] Yu Juan;Zhu Yingxin;OuYang qin;est. Exploratory experimental study on the physiological indexes of human body under the condition of blowing[A]. China building society, HVAC branch, China Refrigeration Association air conditioning heat pump Specialized Committee. National HVAC refrigeration 2010 academic annual conference proceedings [C]. China building society, HVAC branch, China Refrigeration Association air conditioning heat pump Specialized Committee:, 2010:1.(In Chinese)

[12] Xia, Yan; Yung, Yiu-Fai; Zhang, Wei. Evaluating the Selection of Normal-Theory Weight Matrices in the Satorra-Bentler Correction of Chi-Square and Standard Errors [J]. Structural Equation Modeling-A Multidisciplinary Journal, 2016, 23.

[13] Di Zhang,Jiazhong He, Yun Zhao. Kernel Fisher Discriminant Analysis with Locality Preserving for Feature Extraction and Recognition [J]. International Journal of Computational Intelligence Systems,2013,66. 